\title{
Expression of ATP binding cassette E1 enhances viability and invasiveness of lung adenocarcinoma cells in vitro
}

\author{
YE TIAN $^{1}$, XIN TIAN $^{2}$, XU HAN $^{1}$, YONG CHEN $^{1}$, CHENG-YANG SONG $^{1}$, YAN-BIN ZHANG $^{1}$ and DA-LI TIAN ${ }^{1}$ \\ ${ }^{1}$ Department of Thoracic Surgery, The Fourth Affiliated Hospital of China Medical University; \\ ${ }^{2}$ Department of Molecular Oncology, Cancer Research Institute, The First Affiliated Hospital of China Medical University, \\ Shenyang, Liaoning 110032, P.R. China
}

Received April 22, 2015; Accepted February 17, 2016

DOI: $10.3892 / \mathrm{mmr} .2016 .5388$

\begin{abstract}
ATP binding cassette E1 (ABCE1), a member of the family of ATP binding cassette transporters, has initially emerged as an RNase L inhibitor. As a highly conserved protein, it is involved in capsid assembly and translation processes of the human immunodeficiency virus as well as in tumor development and progression. Studies have shown that ABCE1 protein was overexpressed in lung carcinoma tissues and metastatic lymph nodes compared to normal lung tissues. However, little is known about the roles of ABCE1 in lung cancer. The present study investigated the biological effects of vector-mediated ABCE1 overexpression in lung cancer cells in vitro and examined the underlying molecular mechanisms. Overexpression of ABCE1 in the LTEP-a-2 lung adenocarcinoma cell line was achieved by transfection with a plasmid containing full-length ABCE1 cDNA. The ectopic expression of ABCE1 was shown to promote the viability and invasive capacity of lung cancer cells, and to in reduce p27 expression. However, overexpression of ABCE1 did not significantly affect the cell cycle distribution. In conclusion, the present study suggested that ABCE1 promotes the growth, invasion and metastasis of lung adenocarcinoma cells and may represent a potential biomarker and therapeutic target for lung cancer.
\end{abstract}

\section{Introduction}

Lung cancer is the leading cause of cancer-associated mortality worldwide, accounting for 1.38 million mortalities in 2008 (1). Non-small cell lung cancer (NSCLC) constitutes $\sim 85 \%$ of all lung cancer cases and mainly consists of lung adenocarcinoma, squamous cell carcinoma and large cell carcinoma. The current standard treatment modalities for NSCLC, including

Correspondence to: Dr Da-Li Tian, Department of Thoracic Surgery, The Fourth Affiliated Hospital of China Medical University, 4 Chongshan East Road, Shenyang, Liaoning 110032, P.R. China

E-mail: dalitian@hotmail.com

Key words: ABCE1, viability, invasion, lung adenocarcinoma lung adenocarcinoma, include surgery, chemotherapy, radiation therapy and targeted therapy. While surgical resection is an effective treatment option for early-stage NSCLC (2), local recurrence and distant metastases occur in 50-70\% of cases, resulting in an overall five-year survival rate of only $40 \%$ (3). Tumor progression and metastasis are the most common events leading to mortality. At the molecular level, these events are regulated by upregulated oncogenes or de-activation of tumor suppressor genes as a result of genetic mutations, which may either occur spontaneously or be driven by environmental factors or oncogenes. Targeted therapies, such as use of gefitinib, bevacizumab, and crizotinib, directed against genes including EGFR, VEGF and EML4-ALK have been explored for treating advanced cancer; however, the therapeutic outcome has been shown to be restricted by inter-individual variability in drug response and development of drug resistance (4). It is therefore required to identify novel molecular mechanisms and oncogenes responsible for tumor progression and metastasis in order to provide novel approaches or strategies to effectively control lung cancer and improve the prognosis of affected patients.

The present study focused on adenosine triphosphate binding cassette $(\mathrm{ABC})$ transporters, which are a large family of proteins with a variety of functions in cells. This family of proteins can be classified into seven distinct sub-families, designated as ABC A-G (5). ABC transporters are well known to contribute to drug resistance by operating as efflux pumps and their expression shows a significant inter-individual variability, therefore leading to a marked heterogeneity in patient responses to chemotherapy (6). Among these ABCs, ABCE1 protein has initially emerged as an RNase L inhibitor involved in a broad range of biological functions, including response to viral infection, cell proliferation and evasion of apoptosis (7-9). In addition, the $\mathrm{ABCE} 1$ protein also has an important role in the protein translation process $(10,11)$ and multi-drug resistance. Several patient studies by other groups and ours indicated that ABCE1 expression is associated with tumor progression (12-15). The evolutionarily conserved protein ABCE1 has been identified to be essential for eukaryotic cell viability due to having central roles in protein synthesis, particularly in the process of ribosome recycling. Suppression of ABCE1 expression by small interfering (si)RNA was shown to inhibit the proliferation of HEK293 and arrest the growth 
at the gastrula stage of development of Xenopus (10). However, the current status of knowledge on the essential functions of ABCE1 identified using cell and animal models does not explain for its roles in malignant tumors. As the roles of ABCE1 in the growth and metastasis of lung cancer have not been previously elucidated, the present in vitro study assessed the effects of ABCE1 overexpression on the LTEP-a-2 human lung adenocarcinoma cell line. Following transfection of plasmid vector containing full-length ABCE1 complementary (c)DNA, the effects on cell growth, invasive capacity and cell cycle distribution were assessed.

\section{Materials and methods}

Cell line and culture. The LTEP-a-2 human lung adenocarcinoma cell line was obtained from the Cell Bank of the Chinese Academy of Science (Shanghai, China) and cultured in RPMI 1640 medium supplemented with $10 \%$ fetal bovine serum (HyClone, Logan, UT, USA), $100 \mathrm{IU} / \mathrm{ml}$ penicillin and $100 \mu \mathrm{g} / \mathrm{ml}$ streptomycin (Sigma-Aldrich, St. Louis, MO, USA) at $37^{\circ} \mathrm{C}$ in a humidified atmosphere containing $5 \% \mathrm{CO}_{2}$. Cells were grown on sterilized glass Petri dishes and passaged with $0.25 \%$ trypsin (Gibco; Thermo Fisher Scientific, Inc., Waltham, MA, USA).

Construction of ABCE1 cDNA plasmid and stable transfection. For ectopic ABCE1 expression, the enhanced green fluorescence protein plasmid (pEGFP-C1) carrying ABCE1 cDNA was constructed. Briefly, the full-length mRNA of the ABCE1 gene was extracted using the reverse-transcription polymerase chain reaction (RT-PCR) assay. Primers of the full length of ABCE1 were F, 5'-CTCAAGCTTCGATGG CAGACAAGTTAACGAG-3' and R, 5'-CCTGGATCCCTA ATCATCCAAGAAAAAG-3' (Takara Bio, Inc., Dalian, China). The full-length mRNA of ABCE1 and pEGFP-C1 plasmids (BioVector NTCC, Inc. Beijing, China) were digested by Hind III/BamHI (NEB Beijing, Ltd., Beijing, China) and connected by a T4 ligase (NEB Beijing, Ltd.). The pEGFP-ABCE1 recombinant plasmid was then transfected into DH5 $\alpha$ cells (Takara Bio, Inc.) and screened overnight. The recombinant vectors were selected from single colonies and amplified, and confirmed by electrophoresis and DNA sequencing. LTEP-a-2 cells were seeded into six-well plates and incubated for attachment overnight. The pEGFP-ABCE1 plasmid or empty vector were transfected into the cells using Lipofectamin LTX \& PLUS (Invitrogen; Thermo Fisher Scientific, Inc.) following the manufacturer's instructions. After $48 \mathrm{~h}$ of transfection, the cells were cultured in RPMI 1640 medium containing $0.5 \mathrm{mg} / \mathrm{ml} \mathrm{G} 418$ (Gibco) for three weeks and the ABCE1 expression was confirmed in the G418-resistant cell population using RT-PCR and western blot analyses.

RT-PCR. Total RNA of each cell sample was isolated using TRIzol reagent (Takara Bio, Inc.) and reversely transcribed into cDNA by using the PrimeScript RT-PCR kit (Takara Bio, Inc.) according to the manufacturer's instructions. Subsequently, these cDNA samples were subjected to PCR amplification. The primer sequences were as follows: ABCE1 forward, 5'-TCA AACTTCACAGGTTGCC-3' and reverse, 5'-GATCATGTT
CCACCACAATG-3'; glyceraldehyde-3-phosphate dehydrogenase (GAPDH) forward, 5'-GCACCGTCAAGGCTG AGAAC-3' and reverse, 5'-TGGTGAAGACGCCAGTGGA-3' (Takara Bio, Inc.). The composition of the PCR mixture $(10 \mu \mathrm{l})$ was $0.05 \mu \mathrm{l} \mathrm{Taq,} 2 \mu 15 \mathrm{X}$ Buffer, $1 \mu \mathrm{ldNTP}, 5.5 \mu \mathrm{lddH}_{2} 0,0.25 \mu \mathrm{l}$ Primer F, $0.25 \mu \mathrm{l}$ Primer R and $1 \mu \mathrm{l} \mathrm{cDNA}$. The thermocycling conditions used for the PCR Thermal Cycler Dice TP600 (Takara Bio, Inc.) comprised 27 cycles of denaturation at $94^{\circ} \mathrm{C}$ for $30 \mathrm{sec}$, annealing at $58^{\circ} \mathrm{C}$ for $30 \mathrm{sec}$ and extension at $72^{\circ} \mathrm{C}$ for $30 \mathrm{sec}$. After electrophoresis on a $1.5 \%$ agarose gel, images of the PCR bands were captured using a Dolphin-View Image system (Wealtec Corp., Sparks, NV, USA).

Protein extraction and western blot analysis. Total cellular protein was extracted from the cultured cells using lysis buffer containing $50 \mathrm{mM}$ Tris- $\mathrm{HCl}$ (pH 8.0), $150 \mathrm{mM}$ $\mathrm{NaCl}, 0.5 \%$ Nonidet $\mathrm{P} 40,0.5 \%$ sodium deoxycholate and phenylmethylsulfonyl fluoride (Sigma-Aldrich). The protein concentration was determined using the bicinchoninic acid method (Sigma-Aldrich). Aliquots of $100 \mu \mathrm{g}$ protein were then separated by 5 or $10 \%$ sodium dodecyl sulfate polyacrylamide gel electrophoresis for $90 \mathrm{~min}$ and electrotransferred (90 mA, $90 \mathrm{~min}$ ) onto a polyvinylidene fluoride membrane (Millipore, Billerica, MA, USA). The membranes were rinsed with Tris-buffered saline containing Tween-20 (TBS-T; Sigma-Aldrich) and blocked in 5\% non-fat dry milk/TBS-T for $2 \mathrm{~h}$ at room temperature. Subsequently, the membranes were incubated with a rabbit polyclonal anti-ABCE1 (1:400) and p27 (1:200; Santa Cruz Biotechnology, Inc., Dallas, TX, USA; cat. nos. sc-99064 and sc-528, respectively) overnight at $4^{\circ} \mathrm{C}$. Following washing of the membranes three times with TBS-T, they were incubated with a goat anti-rabbit antibody at 1:5,000 dilution for $1 \mathrm{~h}$ The protein bands were then visualized using an enhanced chemiluminescence kit (Thermo Fisher Scientific, Inc.). GAPDH antibody (Santa Cruz Biotechnology, Inc.) was used as a loading control. Subsequently, the membranes were incubated with a rabbit polyclonal anti-ABCE1 (1:400) and p27 (1:200; Santa Cruz Biotechnology, Inc., Dallas, TX, USA; cat. nos. sc-99064 and sc-528, respectively) overnight at $4^{\circ} \mathrm{C}$. Following washing of the membranes three times with TBS-T, they were incubated with a goat anti rabbit antibody at 1:5,000 dilution for $1 \mathrm{~h}$ (Abcam, Cambridge, UK; cat. no. ab6721). The protein bands were then visualized using an enhanced chemiluminescence kit (Thermo Fisher Scientific, Inc.). Subsequently, the same membrane probed for GAPDH protein was eluted and probed with p27 protein (1:200; Santa Cruz Biotechnology, Inc.), and then visualized again.

Cell viability assay. Stably pEGFP-ABCE1-transfected LTEP-a-2 cells were seeded into 96 -well plates $\left(8 \times 10^{3}\right.$ cells/well in $100 \mu \mathrm{l}$ ) were cultured for $2,24,48,72$ or $96 \mathrm{~h}$. The growth medium containing G418 $(0.5 \mathrm{mg} / \mathrm{ml})$ was replaced every three days. At the end of each experiment, $50 \mu 1$ 3-(4,5-dimethylthiazol-2-yl)-2,5-diphenyltetrazolium bromide (MTT; Nanjing KeyGen Biotech Co., Ltd., Nanjing, China) solution was added to each well and the cells were incubated for an additional $4 \mathrm{~h}$. The supernatant was then removed and $150 \mu \mathrm{l}$ dimethyl sulfoxide (Nanjing KeyGen Biotech Co., Ltd.) was added to each well to dissolve the formazan crystals. The optical density was measured using a spectrometer 
(Tecan Sunrise, Tecan, Männedorf, Switzerland) at $570 \mathrm{~nm}$. The experiment was performed in triplicate with two wells for each experimental condition.

Flow cytometric cell cycle analysis. Stably pEGFP-ABCE1-transfected LTEP-a-2 cells were detached with $0.5 \%$ trypsin, washed with cold phosphate-buffered saline (PBS) and fixed with $70 \%$ cold ethanol (Novatech Enterprise Co., Ltd., Jiangsu, China) at $4^{\circ} \mathrm{C}$ overnight. Subsequently, the cells were rehydrated with PBS containing RNase A (Nanjing KeyGen Biotech Co., Ltd.) at $37^{\circ} \mathrm{C}$ for $30 \mathrm{~min}$. Following staining with propidium iodide (Nanjing KeyGen Biotech Co., Ltd.) for $45 \mathrm{~min}$ in the dark, cells were subjected to cell cycle analysis using a flow cytometer (BD FACSCalibur; BD Biosciences, Franklin Lakes, NJ, USA). The percentage of cells in each phase of the cell cycle was determined using the Modfit LT 3.0. software (Verity Software House, Inc., Topsham ME, USA).

Transwell invasion assay. Stably pEGFP-ABCE1-transfected LTEP-a-2 cells were detached with $0.5 \%$ trypsin (Gibco; Thermo Fisher Scientific, Inc., Waltham, MA, USA), washed with cold PBS and counted. For the tumor cell invasion assay, $4 \times 10^{4}$ cells were suspended in serum-free medium and seeded into the upper chambers of Transwell inserts with Matrigel (BD Biosciences)-coated $8-\mu \mathrm{m}$ porous polycarbonate membranes (Corning, Inc., Corning, NY, USA). The lower chambers were then filled with medium containing $10 \%$ fetal bovine serum. After $48 \mathrm{~h}$ of incubation at $37^{\circ} \mathrm{C}$, the cells on the upper surface of the chambers were completely removed using cotton swabs and the cells on the bottom surface that had migrated through the pores were fixed with $4 \%$ paraformaldehyde (Novatech Enterprise Co., Ltd.) for $30 \mathrm{~min}$ and stained with crystal violet (Sigma-Aldrich). The experiments were performed in triplicate wells and repeated at least once. To quantify tumor cell invasion, 10 high-power microscopic fields (magnification, $\mathrm{x} 400$ ) were counted and averaged for comparison to the control cells using the IX71 microscope with a DP70 Color Camera (Olympus Corporation, Tokyo, Japan).

Statistical analysis. Values are expressed as the mean \pm standard deviation. All statistical calculations were performed using SPSS software, version 17.0 for windows (SPSS, Inc., Chicago, IL, USA). Differences for each experimental group were compared using one-way analysis of variance. $\mathrm{P}<0.05$ was considered to indicate a statistically significant difference.

\section{Results}

Ectopic expression of ABCE1 in lung adenocarcinoma cells. First, the pEGFP-ABCE1 vector was constructed and stably transfected into LTEP-a-2 cells. The induced expression of ABCE1 mRNA and protein was confirmed by RT-PCR and western blot analysis, respectively. As shown in Fig. 1A, transfection with pEGFP-ABCE1 recombinant plasmid markedly increased the expression of ABCE1 mRNA in LTEP-a-2 cells. Furthermore, western blot analysis showed that the expression of ABCE1 protein was also increased compared to that in the parental and empty vector-transfected cells (Fig. 1B). The presence of a $\sim 95 \mathrm{KDa}$ recombinant protein corresponded to the fusion of the $\sim 27 \mathrm{KDa}$ EGFP and the $\sim 68 \mathrm{KDa} \mathrm{ABCE} 1$ protein.

Overexpression of $A B C E 1$ results in reduced p27 expression in lung adenocarcinoma cells. To explore the effects of ABCE1 in LTEP-a-2 cells, the expression of p27 was assessed using western blot analysis. As shown in Fig. 1C, p27 protein levels were markedly reduced in cells overexpressing ABCE1 compared to that in the parental cells and empty vector-transfected cells.

Overexpression of $A B C E 1$ enhances the proliferation of lung adenocarcinoma cells. Next, the present study determined the effects of the induced ABCE1 expression on the proliferation of LTEP-a-2 cells in vitro. Cells transfected with pEGFP-ABCE1, empty vector or parental cells were cultivated for $2,24,48,72$ or $96 \mathrm{~h}$ and quantified using an MTT assay. The growth curves revealed that the proliferation of LTEP-a-2 cells transfected with pEGFP-ABCE1 was significantly enhanced to $119.63,126.55,121.58$ and $128.13 \%$ of that of the control cells after 24, 48, 72 and $96 \mathrm{~h}$, respectively $(\mathrm{P}<0.05)$ (Fig. 2). However, the proliferative rates of the parental cells (Control) and empty vector-transfected cells (Mock) were similar ( $\mathrm{P}>0.05)$. These results clearly indicated that $\mathrm{ABCE} 1$ promoted the growth of lung adenocarcinoma cells.

ABCE1 does not affect the cell cycle of lung adenocarcinoma cells. To further determine the mechanisms by which ABCE1 enhances the proliferation of lung adenocarcinoma cells, the cell cycle distribution of LTEP-a-2 cells stably transfected with pEGFP-ABCE1 was assessed by flow cytometry. As shown in Fig. 3, overexpression of ABCE1 did not affect the cell cycle distribution of LTEP-a-2 cells compared with that of empty vector-transfected and parental cells.

ABCE1 expression promotes lung adenocarcinoma cell invasion. The effects of the ABCE1 overexpression on the invasive capacity of LTEP-a-2 cells were assessed using a Transwell assay. After $48 \mathrm{~h}$ of incubation, the number of cells transgressed through the Matrigel-coated membrane was significantly higher in the ABCE1 overexpression group (93 \pm 5 ; $\mathrm{P}<0.05)$ compared with that in the empty vector-transfected group (38 \pm 4 ) and parental cells $(37 \pm 5)$ (Fig. 4). This result demonstrated that ABCE1 enhances the invasive capacity of lung adenocarcinoma cells.

\section{Discussion}

The present study determined the effects of ABCE1 overexpression on the proliferation and invasiveness of lung adenocarcinoma in vitro. The results showed that ectopic expression of ABCE1 promoted tumor cell growth and invasion, and reduced the expression of p27 protein in LTEP-a-2 cells. However, flow cytometric analysis did not show any effect of ABCE1 overexpression on the cell cycle distribution of LTEP-a-2 cells. Overall, these results demonstrated that ABCE1 exerts tumor-promoting effects in lung adenocarcinoma cells.

Tumor progression and metastasis are important factors that contribute to cancer mortality and poor survival of 

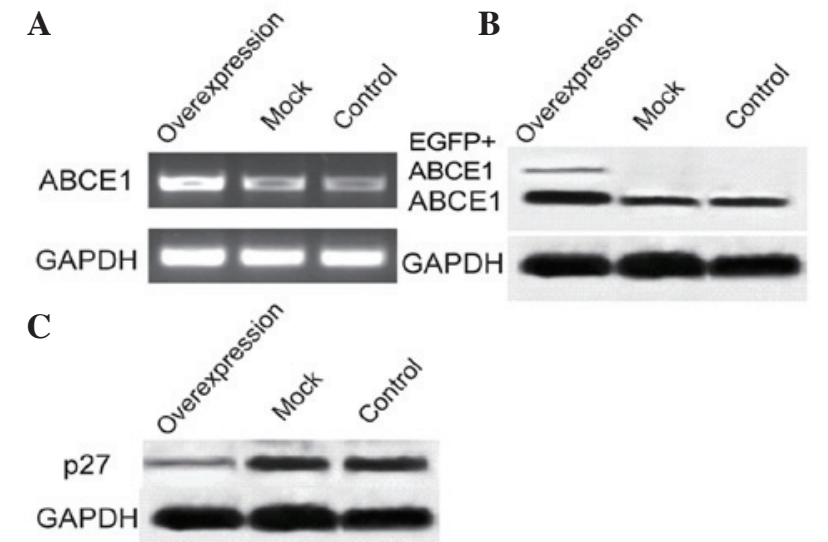

Figure 1. Induction of ABCE1 expression and regulation of p27 expression after transfection of pEGFP-ABCE1. ABCE1 overexpression in LTEP-a-2 lung adenocarcinoma cells stably transfected with ABCE1 expression vector was confirmed using (A) reverse-transcription polymerase chain reaction and (B) western blot analysis. The ABCE1 antibodies were specific for the $95 \mathrm{kDa}$ and $68 \mathrm{kDa}$ proteins of ABCE1. (C) Overexpression of ABCE1 led to a reduction of $\mathrm{p} 27$ protein expression, as indicated by western blot analysis. Overexpression, cells transfected with pEGFP-ABCE1; Mock, cells transfected with empty vector; Control, parental cells; EGFP, enhanced green fluorescence protein; $\mathrm{ABCE} 1$, adenosine triphosphate binding cassette E1; GAPDH, glyceraldehyde-3-phosphate dehydrogenase.

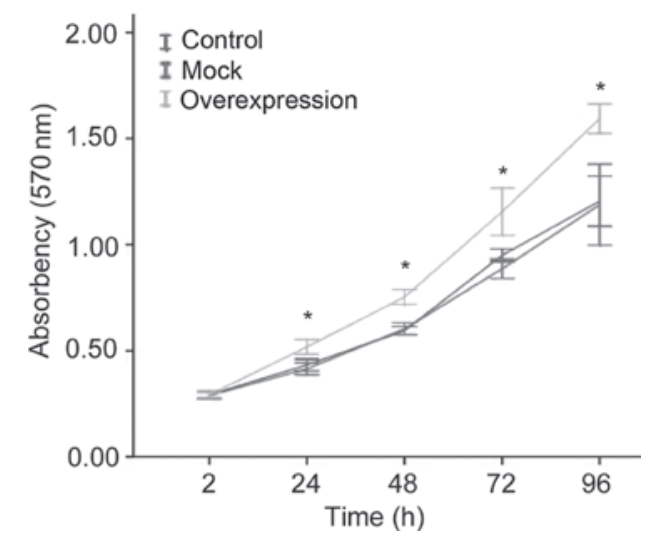

Figure 2. ABCE1 overexpression enhances the proliferation of LTEP-a-2 cells. Growth curves determined by MTT assays are shown. Values are expressed as the mean \pm standard deviation. Experiments were performed in triplicate with two wells per experimental condition each. ${ }^{*} \mathrm{P}<0.05$ vs. mock and control groups. Overexpression, cells transfected with ABCE1 overexpression plasmid; Mock, cells transfected with empty vector; Control, parental cells; ABCE1, adenosine triphosphate binding cassette E1.

cancer patients. During tumor progression and development of metastasis, tumor cells acquire specific properties that enable them to overcome barriers existing in normal tissues. The ABCE1 gene is located on chromosome 4q31 in humans, and its protein is abundant in the cytoplasm and mitochondria (16). It has been reported that ABCE1 is highly expressed in numerous tumor types, including colon cancer (14), hepatocellular carcinoma (15), breast cancer (17) and oral cancer (18). In addition, a previous study by our group demonstrated that expression of ABCE1 protein was associated with advanced clinical stages of lung cancer and tumor lymph node metastasis (13). A recent study by our group showed that ectopic ABCE1 expression promoted the clonogenicity and anchorage-independent growth of
LTEPa-2 cells, had an augmentative effect on tumor growth and metastasis in a mouse xenograft tumor model and reduced the expression of the tumor-suppressor gene growth arrest and DNA damage-inducible $45 \alpha$ (19). As a highly conserved protein, ABCE1 is universally found in eukaryotes and archaeans. Therefore, it was suggested to have an essential role in eukaryote development and translational processes (10). ABCE1 protein has been demonstrated have a central role in the essential process of protein synthesis, particularly in the process of ribosome recycling $(20,21)$. Targeted silencing of the ABCE1 gene in normal cells results in severe physiological dysfunction. Suppression of ABCE1 expression by siRNA was shown to inhibit the proliferation of HEK293 cells and cause growth arrest at the gastrula stage of development in Xenopus (10). This observation was in accordance with the findings of a recent study, which demonstrated that downregulation of ABCE1 protein caused defects in the recognition of a stop codon (22). Consequently, the alter phenotype lung cancer cells obtained by removal of ABCE1 makes it difficult to accurately prove its biological function in malignant tumors. Based on this, the present study assessed the effects of vector-mediated overexpression of ABCE1 in LTEP-a-2 cells, revealing that ABCE1 enhanced their proliferation and invasive capacity in vitro. These observations indicated that ABCE1 may be involved in the development and progression of lung cancer. Consistent with this notion, the gene copy number of ABCE1 has been reported to be elevated in lung adenocarcinoma, colon cancer and ovarian cancer cells (23).

A previous microarray analysis by our group revealed that a number of genes were differentially expressed after ABCE1 knockdown (13). Among these, p27 was selected for further study of the mechanisms induced by ABCE1 in lung adenocarcinoma in the present study. Overexpression of endogenous ABCE1 in LTEP-a-2 cells was found to result in a reduced expression of $\mathrm{p} 27$ protein, which confirmed the result of our previous study. P27 protein is a well-characterized tumor suppressor and is frequently inactivated in a large variety of cancer types with increased aggressiveness and poor clinical outcome $(24,25)$. In NSCLC, low levels of p27 protein are associated with cancer development and poor prognosis (26). Numerous studies have shown that $\mathrm{p} 27$ protein has a dual function in suppressing cell proliferation and affecting motility and invasiveness (27-29). In accordance with these findings, the present study confirmed that overexpression of ABCE1 promoted lung cancer cell proliferation and invasion, directly or indirectly by downregulation of p27 protein. The cell cycle was not demonstrated to be directly affected by the p 27 protein; however, this protein serves a central role in regulating the $\mathrm{Cdk}$ activity in cell progression from the G1 towards the $\mathrm{S}$ phase, and further are required to confirm this fact.

Furthermore, previous studies have reported that ABCE1 not only appeared to negatively regulate the 2-5A/RNase L-associated apoptotic pathway, but also promoted translational processes in eukaryotes (7-11). However, unexpectedly, the results of the present study indicated that ABCE1 overexpression did not affect the cell cycle distribution of LTEP-a-2 cells, which is not in line with the findings of enhanced proliferation and downregulation 

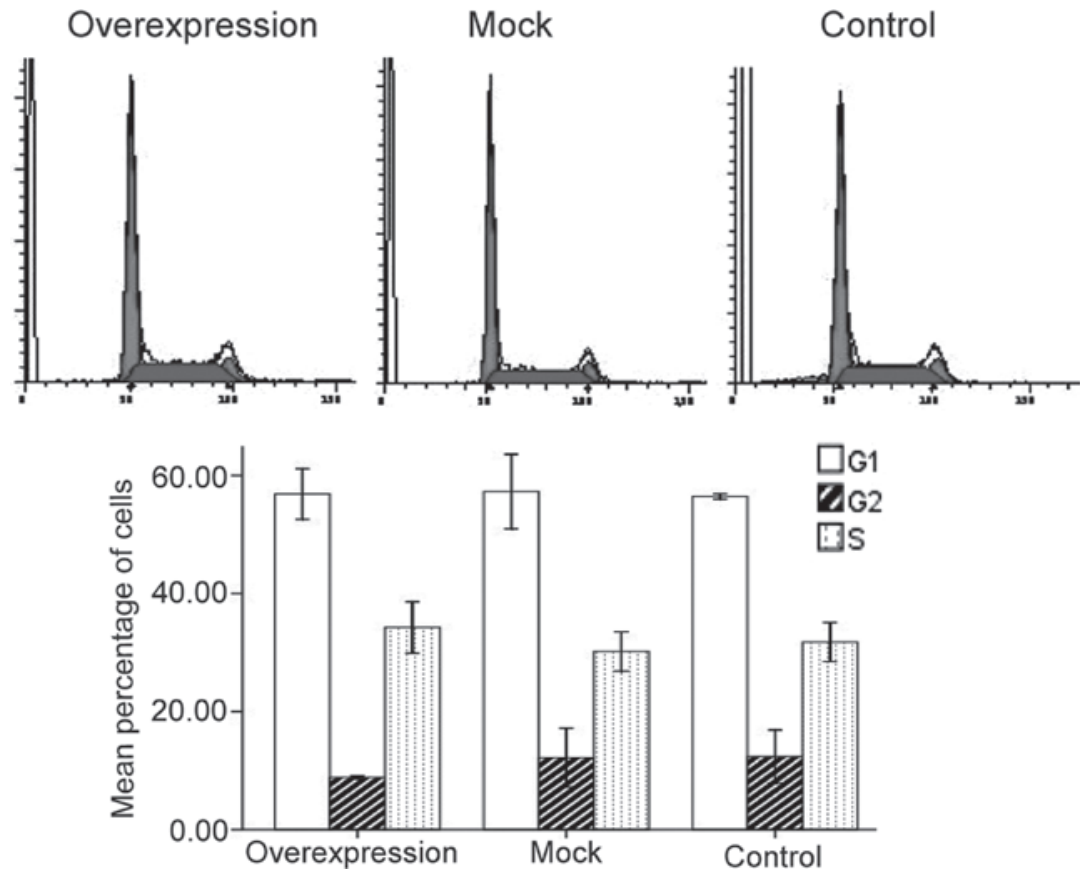

Figure 3. Effects of ABCE1 overexpression on the cell cycle distribution of LTEP-a-2 cells. Following transfection for $72 \mathrm{~h}$, cells were stained with propidium iodide and subjected to flow cytometric analysis. Values are expressed as the mean \pm standard deviation of three independent experiments. Overexpression, cells transfected with ABCE1 overexpression plasmid; Mock, cells transfected with empty vector; Control, parental cells; ABCE1, adenosine triphosphate binding cassette E1.
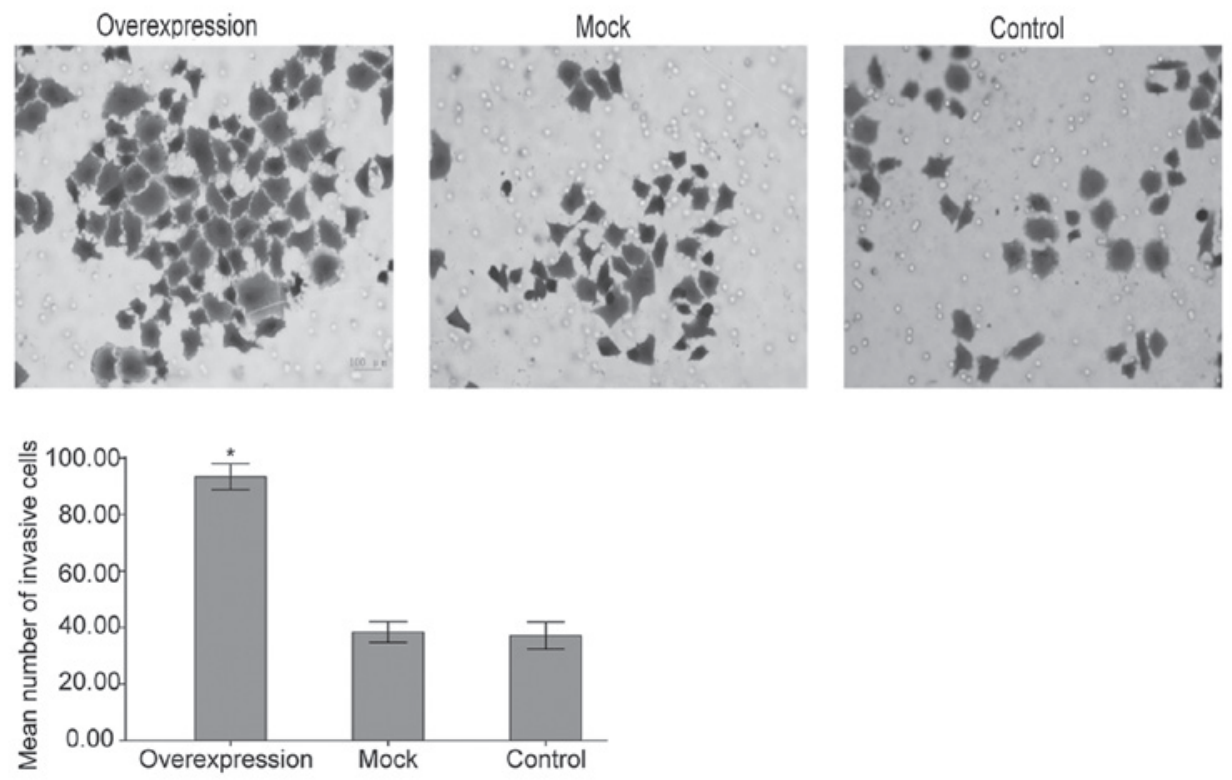

Figure 4. ABCE1 overexpression enhances the invasiveness of LTEP-a-2 cells. A Transwell invasion assay showed that the number of invasive cells in the overexpression group was significantly increased compared to that in the mock and control groups. Representative images of crystal violet-stained cells on the lower surface of the membrane are shown (magnification, $\mathrm{x} 400$ ). Values are expressed as the mean \pm standard deviation of ten microscopic fields for each group. "P $<0.05$ vs Mock/Control group. Overexpression, cells transfected with ABCE1 overexpression plasmid; Mock, cells transfected with empty vector; Control, parental cells; ABCE1, adenosine triphosphate binding cassette E1.

of p27 following ectopic expression of ABCE1. Therefore, it remains to be fully elucidated how ABCE1 contributes to tumor progression.

The $\mathrm{ABCE} 1$ protein is an $\mathrm{ABC}$ transporter whose physiological function has not been well characterized; ABC transporters are widely and constitutively expressed in various cell types other than cancer cells. Functionally, ABC transporters bind to and hydrolyze ATP to transport various molecules across the plasma membrane as well as to intracellular membranes of the endoplasmic reticulum, peroxisome and mitochondria. $\mathrm{ABC}$ transporters are thought to participate in the absorption and secretion of endogenous and exogenous substances (5,7-11). Altered expression of these genes contributes to various types of disease, including cancer (13-16). The observations of the 
present study supported the hypothesis that increased ABCE1 expression is critical in lung cancer progression. Apart from this role, several lines of evidence suggested that ABCE1 is a primary cellular target responsible for the attenuation of damage through reactive oxygen species in diverse aerobic organisms $(30,31)$. This reactive oxygen species-mediated damage is linked to various diseases, including cancer. It is likely that normal levels of ABCE1 are required to maintain healthy cells, while aberrant expression may impact cell fate to possibly drive tumorigenesis and cancer progression. It is therefore essential to determine whether the increased expression of ABCE1 has a significant effect on lung cancer progression, or whether a lower expression leads to increased ROS damage, and possibly carcinogenesis.

The present study reported on the effects of ABCE1 overexpression on lung adenocarcinoma cells in vitro, demonstrating its enhancing effect on cell proliferation and invasiveness with simultaneous downregulation of p27 protein expression. However, further study is required to more precisely define the exact underlying mechanisms of the observed effects of ABCE1 in NSCLCs.

\section{Acknowledgements}

The present study was supported by the Young Innovation and Development Foundation of the Fourth Affiliated Hospital of China Medical University and the National Nature Science Foundation of China (no. 30973502).

\section{References}

1. Ferlay J, Shin HR, Bray F, Forman D, Mathers C and Parkin DM: Estimates of worldwide burden of cancer in 2008: GLOBOCAN 2008. Int J Cancer 127: 2893-2917, 2010.

2. Chemotherapy in non-small cell lung cancer: A meta-analysis using updated data on individual patients from 52 randomised clinical trials. Non-small cell lung cancer collaborative group. BMJ 311: 899-909, 1995.

3. Filipits M, Pirker R, Dunant A, Lantuejoul S, Schmid K, Huynh A, Haddad V, André F, Stahel R, Pignon JP, et al: Cell cycle regulators and outcome of adjuvant cisplatin-based chemotherapy in completely resected non-small-cell lung cancer: The international adjuvant lung cancer trial biologic program. J Clin Oncol 25: 2735-2740, 2007.

4. Mamdani H, Induru R and Jalal SI: Novel therapies in small cell-lung cancer. Transl Lung Cancer Res 4: 533-544, 2015.

5. Dean M, Hamon Y and Chimini G: The human ATP-binding cassette $(A B C)$ transporter superfamily. J Lipid Res 42: 1007-1017, 2001.

6. Mendoza JL, Urcelay E, Lana R, Martín MC, López N, Guijarro LG, Mayol JA, Taxonera C, de la Concha EG, Peña AS and Díaz-Rubio M: MDR1 polymorphisms and response to azathioprine therapy in patients with Crohn's disease. Inflamm Bowel Dis 13: 585-590, 2007.

7. Bisbal C, Martinand C, Silhol M, Lebleu B and Salehzada T: Cloning and characterization of a RNase $\mathrm{L}$ inhibitor. A new component of the interferon-regulated 2-5 A pathway. J Biol Chem 270: 13308-13317, 1995.

8. Hassel BA, Zhou A, Sotomayor C, Maran A and Silverman RH: A dominant negative mutant of 2-5A-dependent RNase suppresses antiproliferative and antiviral effects of interferon. EMBO J 12: 3297-3304, 1993.

9. Le Roy F, Bisbal C, Silhol M, Martinand C, Lebleu B and Salehzada T: The 2-5A/RNase L/RNase L inhibitor (RLI) [correction of (RNI)] pathway regulates mitochondrial mRNAs stability in interferon alpha-treated H9 cells. J Biol Chem 276 : 48473-48482, 2001
10. Chen ZQ, Dong J, Ishimura A, Daar I, Hinnebusch AG and Dean M: The essential vertebrate ABCE1 protein interacts with eukaryotic initiation factors. J Biol Chem 281: 7452-7457, 2006.

11. Pisareva VP, Skabkin MA, Hellen CU,Pestova TV and Pisarev AV: Dissociation by Pelota, Hbs1 and ABCE1 of mammalian vacant 80S ribosomes and stalled elongation complexes. EMBO J 30: 1804-1817, 2011.

12. Huang B, Gao Y, Tian D and Zheng M: A small interfering ABCE1-targeting RNA inhibits the proliferation and invasiveness of small cell lung cancer. Int J Mol Med 25: 687-693, 2010.

13. Ren Y, Li Y and Tian D: Role of the ABCE1 gene in human lung adenocarcinoma. Oncol Rep 27: 965-970, 2012.

14. Hlavata I, Mohelnikova-Duchonova B, Vaclavikova R, Liska V, Pitule P, Novak P, Bruha J, Vycital O, Holubec L, Treska V, et al: The role of $\mathrm{ABC}$ transporters in progression and clinical outcome of colorectal cancer. Mutagenesis 27: 187-196, 2012.

15. Furuta M, Kozaki KI, Tanaka S, Arii S, Imoto I and Inazawa $\mathrm{J}$ : MiR-124 and miR-203 are epigenetically silenced tumor-suppressive microRNAs in hepatocellular carcinoma. Carcinogenesis 31: 766-776, 2010.

16. Tian Y, Han X and Tian DL: The biological regulation of ABCE1. IUBMB Life 64: 795-800, 2012.

17. Huang B, Zhou H, Lang X and Liu Z: SiRNA-induced ABCE1 silencing inhibits proliferation and invasion of breast cancer cells. Mol Med Rep 10: 1685-1690, 2014.

18. Wang L, Zhang M and Liu DX: Knock-down of ABCE1 gene induces G1/S arrest in human oral cancer cells. Int J Clin Exp Pathol 7: 5495-5504, 2014.

19. Tian Y, Tian X, Han X, Chen Y, Song CY, Jiang WJ and Tian DL: $\mathrm{ABCE} 1$ plays an essential role in lung cancer progression and metastasis. Tumor Biol: Jan 5, 2016 (Epub ahead of print).

20. Nürenberg E and Tampé R: Tying up loose ends: Ribosome recycling in eukaryotes and archaea. Trends Biochem Sci 38: 64-74, 2013

21. Franckenberg S, Becker T and Beckmann R: Structural view on recycling of archaeal and eukaryotic ribosomes after canonical termination and ribosome rescue. Curr Opin Struct Biol 22: 786-796, 2012.

22. Khoshnevis S, Gross T, Rotte C, Baierlein C, Ficner R and Krebber H: The iron-sulphur protein RNase L inhibitor functions in translation termination. EMBO Rep 11: 214-219, 2010.

23. Yasui K, Mihara S, Zhao C, Okamoto H, Saito-Ohara F, Tomida A, Funato T, Yokomizo A, Naito S, Imoto I, et al: Alteration in copy numbers of genes as a mechanism for acquired drug resistance. Cancer Res 64: 1403-1410, 2004.

24. Slingerland $\mathbf{J}$ and Pagano M: Regulation of the cdk inhibitor p27 and its deregulation in cancer. J Cell Physiol 183: 10-17, 2000.

25. Chu IM, Hengst L and Slingerland JM: The Cdk inhibitor p27 in human cancer: Prognostic potential and relevance to anticancer therapy. Nat Rev Cancer 8: 253-267, 2008.

26. Esposito V, Baldi A, De Luca A, Groger AM, Loda M, Giordano GG, Caputi M, Baldi F, Pagano M and Giordano A: Prognostic role of the cyclin-dependent kinase Inhibitor p27 in non-small cell lung cancer. Cancer Res 57: 3381-3385, 1997.

27. Chen J, Willingham T, Shuford $M$ and Nisen PD: Tumor suppression and inhibition of aneuploid cell accumulation in human brain tumor cells by ectopic overexpression of the cyclin-dependent kinase inhibitor p27KIP1. J Clin Invest 97: 1983-1988, 1996.

28. Díez-Juan A and Andrés V: Coordinate control of proliferation and migration by the p27kip1/cyclin-dependent kinase/retinoblastoma pathway in vascular smooth muscle cells and fibroblasts. Circ Res 92: 402-410, 2003.

29. Supriatno, Harada K, Kawaguchi S, Yoshida H and Sato M: Effect of p27Kip1 on the ability of invasion and metastasis of an oral cancer cell line. Oncol Rep 10: 527-532, 2003.

30. Alhebshi A, Sideri TC, Holland SL and Avery SV: The essential iron-sulfur protein Rli1 is an important target accounting for inhibition of cell growth by reactive oxygen species. Mol Biol Cell 23: 3582-3590, 2012.

31. Zhai C, Li Y, Mascarenhas $\mathrm{C}$, et al: The function of ORAOV1/LTO1, a gene that is overexpressed frequently in cancer: Essential roles in the function and biogenesis of the ribosome. Oncogene 33: 484-494, 2014. 